\title{
Stability regime of pulse frequency for single event electrospraying
}

\author{
Urszula Stachewicz, ${ }^{1, a)}$ Caner U. Yurteri, ${ }^{1}$ Jan C. M. Marijnissen, ${ }^{1}$ and J. Frits Dijksman ${ }^{2}$ \\ ${ }^{1}$ DelftChemTech, Delft University of Technology, Delft 2628 BL, The Netherlands \\ ${ }^{2}$ Philips Research Europe, Eindhoven 5656 AE, The Netherlands
}

(Received 1 September 2009; accepted 8 November 2009; published online 3 December 2009)

\begin{abstract}
This study demonstrates that the hydrodynamic phenomena taking place inside the capillary limits the frequency range of voltage pulses for stable single event electrospraying (SEE), when the meniscus/cone size is defined by the inner diameter of the nozzle. For the analysis of SEE, we used a one-dimensional model describing the displacement and oscillations of the liquid inside the nozzle. The frequency range of voltage pulses for stable SEE is related to the natural oscillation frequency of the fluid column inside the capillary. (C) 2009 American Institute of Physics.
\end{abstract}

[doi:10.1063/1.3269594]

Electrohydrodynamic processes have been used for the dispensing of picoliters or smaller spots in patterning techniques ${ }^{1-3}$ and for the deposition of a wide range of materials for applications in electronics ${ }^{4,5}$ and analytical chemistry. ${ }^{3,5,6}$ In electrospraying, a potential is applied between a capillary and a grounded plate separated at a certain distance. Due to the high field strength, the meniscus changes into a cone and the liquid starts to emerge from the tip of the cone as a thin jet, which breaks up into fine droplets. The charged droplets are accelerated in the electric field ${ }^{7}$ having its highest strength near the tip of the cone. ${ }^{8}$ Moreover, the application of ac pulses onto a bias voltage with well adjusted frequency gives a possibility to control the droplet size with the given flow rate. ${ }^{9,10}$ For example, this method was used to show the influence of the voltage pulse frequency $(5-25 \mathrm{~Hz})$ onto the deposited droplet size during electrospraying of ethylene glycol with methylene blue. ${ }^{11}$ Pulses of $1 \mathrm{kV}$ were applied on the bias voltage of $2 \mathrm{kV}$ and the distance between the stainless steel nozzle and the counterelectrode was $1 \mathrm{~mm}$. In those experiments, the flow rate was controlled with a syringe pump and the frequency of the voltage pulses was tuned to the frequency of the intermittent mode. It was reported that, for frequencies higher than 25 $\mathrm{Hz}$, it was not possible to spray in the cone-jet mode anymore.

In order to use electrospraying as an on-demand deposition method, well defined and reproducible single event electrospraying (SEE) have been studied. We generated stable and short events of jetting for each applied pulse. ${ }^{12}$ The refilling of the fluid that has left the system during SEE took place autonomously driven by surface tension and gravity. So far, we demonstrated in our studies that, with SEE, we can deposit a few picoliters of liquid when the meniscus and the cone size are confined to the inner diameter (ID) of the capillary. In this study also $50 \mu \mathrm{m}$ nozzle was used. ${ }^{13}$ Furthermore, a model describing the one-dimensional motion of the liquid in the capillary was developed and allowed us to calculate the electrodynamic and hydrodynamic relaxation times involved in SEE, such as the refilling time, the time needed for the liquid inside the capillary to return from the disturbed to the equilibrium situation. ${ }^{14,15}$ Continuing our research on using electrospraying as an on-demand deposition method, it is important to understand the influence of the frequency of the voltage pulses on SEE. Only then, SEE can be used as a reproducible on-demand deposition method. Therefore, in this study we want to address the characteristics of SEE in the frequency domain. We determine theoretically the frequency range for stable SEEs by carefully examining the hydrodynamic times involved and confirm it with the experimental results.

In our previous work, we proposed a one-dimensional model for the analysis of the characteristic times involved in SEE. ${ }^{14}$ Included in this model are electrostatics and electrodynamics on the one hand, and hydrostatic, fluid dynamic, and surface tension effects on the other hand. To calculate the hydrodynamic times involved in SEE we start with the description of the capillary system in a static equilibrium situation. In Fig. 1, the glass capillary of our electrospraying setup, when no voltage is applied, is presented. In the equilibrium situation, $P_{\mathrm{fc}}$, the static pressure of the liquid column, $P_{\mathrm{mc}}$, the capillary pressure of the meniscus inside the capillary far above the thinnest part of the nozzle and $P_{\mathrm{m}}$, the capillary pressure caused by the liquid meniscus protruding beyond the end of the nozzle have to be taken into account. For the static equilibrium situation inside the capillary, the pressure balance at the nozzle front is $P_{\mathrm{fc}}+P_{\mathrm{m}}-P_{\mathrm{mc}}=0$.

The calculation of the meniscus pressure is based on the meniscus height measurement just before the SEE generation, when no voltage is applied. With the Young-Laplace relation, the pressure caused by the meniscus of the fluid hanging outside the capillary can be calculated, $P_{\mathrm{m}}=2 \sigma / r_{\mathrm{c}}$, where $\sigma$ is surface tension and $r_{\mathrm{c}}$ is the meniscus radius of curvature, which follows directly from the meniscus height $h_{\mathrm{m}}, r_{\mathrm{c}}=\left(h_{\mathrm{m}}^{2}+r_{\mathrm{n}}^{2}\right) /\left(2 h_{\mathrm{m}}\right)$, where $r_{\mathrm{n}}$ is the inner capillary radius to which the meniscus is attached. The equation describing the electrohydrodynamic movements of the liquid column in the capillary is similar to the equation describing damped free oscillations ${ }^{16}$

$$
m \ddot{x}+c \dot{x}+k x=0,
$$

where $m$ is the mass, $c$ is the damping coefficient, $k$ is the spring constant, and $x$ is the liquid displacement inside the capillary. The components of Eq. (1) are ${ }^{14}$

$$
\begin{aligned}
& m=\pi r_{\mathrm{n}}^{2} \rho_{\mathrm{s}} H, \\
& c=8 \pi \eta l,
\end{aligned}
$$

${ }^{a)}$ Electronic mail: u.stachewicz@tudelft.nl. 


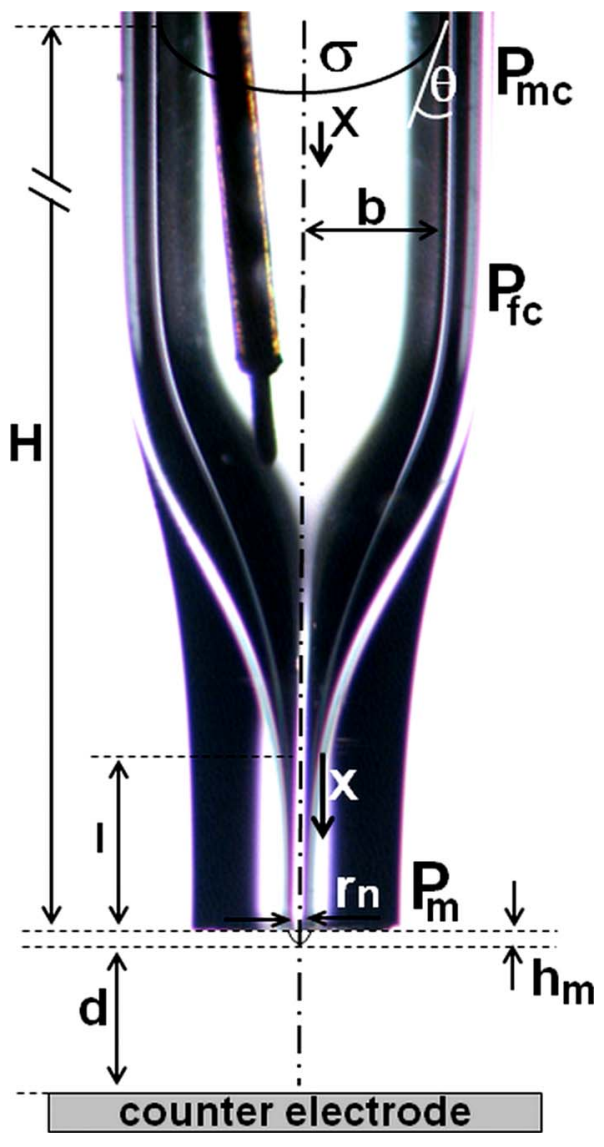

FIG. 1. (Color online) Schematic drawing of the capillary dimensions (cross section) based on a photograph taken with an optical microscope.

$$
k=\frac{8 \pi \sigma r_{\mathrm{n}}^{4}}{\left(r_{\mathrm{n}}^{2}+h_{\mathrm{m}}^{2}\right)^{2}}\left(1-\frac{2 h_{\mathrm{m}}^{2}}{\left(r_{\mathrm{n}}^{2}+h_{\mathrm{m}}^{2}\right)}\right)+\rho_{\mathrm{s}} g \pi \frac{r_{\mathrm{n}}^{4}}{b^{2}},
$$

where $H$ is the total liquid height in the capillary, $l$ is the total length of the thinnest part $(240 \mu \mathrm{m})$, the nozzle, $\eta$ is the viscosity, $\rho_{\mathrm{s}}$ is the density of the solution, $g$ is the acceleration due to gravity, $b$ is the radius of the capillary far above the nozzle $(0.5 \mathrm{~mm})$, and $r_{\mathrm{n}}$ is the radius of the inside of the nozzle. The solution of Eq. (1) yields to the frequency, $f_{\mathrm{c}}$ and damping ratio, $\Gamma_{\mathrm{c}}$ of the liquid inside the capillary as well as the time needed for refilling the liquid inside the capillary, $\tau_{\mathrm{rf}}$

$$
\begin{aligned}
f_{\mathrm{c}} & =\frac{1}{2 \pi} \sqrt{\frac{k}{m}}, \\
\Gamma_{\mathrm{c}} & =\frac{c}{2 \sqrt{k m}}, \\
\tau_{\mathrm{rf}} & =\frac{8 \eta l}{\left[\frac{8 \sigma_{\mathrm{m}} r_{\mathrm{n}}^{4}}{\left(r_{\mathrm{n}}^{2}+h_{\mathrm{m}}^{2}\right)^{2}}\left(1-\frac{2 h_{\mathrm{m}}^{2}}{\left(r_{\mathrm{n}}^{2}+h_{\mathrm{m}}^{2}\right)}\right)+\rho_{\mathrm{s}} g \frac{r_{\mathrm{n}}^{4}}{b^{2}}\right]} .
\end{aligned}
$$

The liquid displacement in the capillary can be over-damped $\left(\Gamma_{\mathrm{c}}>1\right)$ or underdamped $\left(\Gamma_{\mathrm{c}}<1\right)$. In case the damping ratio is smaller than $1, \Gamma_{\mathrm{c}}<1$, the frequency of the oscillating liquid inside the capillary, $f_{c}$, could influence the SEE process. When the ID of the nozzle is very small, the refilling time is very short. Therefore, we assume that the limiting
TABLE I. Experimental settings for SEE with modified capillaries with the dimension of the capillary and meniscus, and characteristic values.

Parameter

Bias voltage. $U_{\mathrm{b}}(\mathrm{V})$ 0

Pulse voltage, $U_{\mathrm{p}}(\mathrm{V})$

3000

Pulse time, $t_{\mathrm{p}}(\mathrm{ms})$

0.5

Distance between capillary and counter electrode,

$d+h_{\mathrm{m}}(\mathrm{mm})$

Approximate liquid column height, $H(\mathrm{~m})$

$15 \times 10^{-2}$

Meniscus height, $h_{\mathrm{m}}(\mathrm{m})$

Meniscus curvature radius, $r_{\mathrm{c}}(\mathrm{m})$

$10 \times 10^{-6}$

Refill time, $\tau_{\mathrm{rf}}(\mathrm{s})$

$3.63 \times 10^{-5}$

Frequency of liquid oscillations inside the capillary,

$f_{\mathrm{c}}(\mathrm{Hz})$

$49.2 \times 10^{-6}$

Damping ratio in capillary, $\Gamma_{\mathrm{c}}(1 / \mathrm{s})$

hydrodynamic effect can be related to the natural oscillation frequency of the liquid inside the capillary.

The experiments have been performed with the modified glass capillary (Autodrop AD-H-501 Microdrop Technologies, Norderstedt, Germany), with outer diameter of $600 \mu \mathrm{m}$ and ID of $50 \mu \mathrm{m}$. A high voltage was supplied to the liquid inside the glass capillary by a metal wire inside it. The modified capillary has an antiwetting coating on the outer surface of the glass capillary, close to the orifice. This coating defines the attachment of the meniscus to the inside rim of the nozzle's orifice. ${ }^{14}$ The experiments were performed with a solution of ethylene glycol (Merck, purity $\mathrm{GC} \geq 99.5$ ) and deionized water, in the proportion of $70 / 30 \%(\mathrm{v} / \mathrm{v})$, respectively, that had a surface tension $\sigma=55.4 \mathrm{mN} / \mathrm{m}$, viscosity $\eta=6.11 \mathrm{mPa} \cdot \mathrm{s}$, density $\rho_{\mathrm{s}}=1078.64 \mathrm{~kg} / \mathrm{m}^{3}$, and conductivity $\kappa=2.7 \times 10^{-4} \mathrm{~S} / \mathrm{m}$. All experiments were recorded using a charge coupled device camera (Jai CV-M10-SX) with a microscopic objective (Optem 70XL 29-20-39) and a stroboscope with adjustable flash delay (LumiLED, LUXEON). The image system was triggered by the leading edge of the pulse. The adjustable flash delay enabled us to take pictures of different moments in time, during, and after the applied pulse. By concatenation of all images, taken here with the consecutive flash delay of $5 \mu \mathrm{s}$, we were able to record the whole SEE. Based on the recorded SEE pictures the ejection time was determined. The ejection time is defined from the moment when the first visible droplet was ejected from the just formed cone to the instant, where the ejection of droplets was not observed anymore.

SEEs were generated with $500 \mu$ s rectangular pulses of $3 \mathrm{kV}$, applied without a bias voltage, $\left(U_{\mathrm{b}}=0\right)$. For all experiments we kept the same settings and changed only the frequency of pulses from 10 to $1000 \mathrm{~Hz}$, Table I. As shown in Fig. 2, the ejection time was constant till approximately 450 Hz. For higher frequencies, first the ejection time reduced and intermittent ejections were observed. As shown in Fig. 3, the cone-jet mode was pulsating and the ejection of the liquid from the cone became unpredictable. Above $750 \mathrm{~Hz}$, there was almost no ejection of the liquid anymore. Moreover, by increasing the pulse frequency, $f_{\mathrm{p}}$, the starting point of ejections is slightly delayed. The capillary dimensions and calculated characteristic values are presented in Table I. Those results reveal that the system here presented was under-damped $\left(\Gamma_{\mathrm{c}}<1\right)$ and the frequency of the liquid oscillations inside the capillary, $f_{c}$, was $244 \mathrm{~Hz}$. The liquid ejec- 


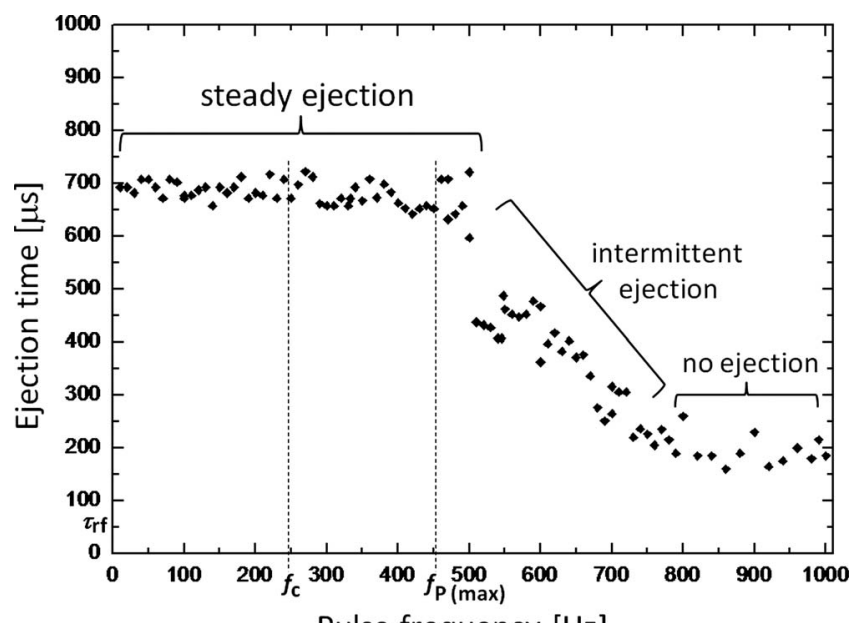

Pulse frequency $[\mathrm{Hz}]$

FIG. 2. Relation between ejection time and the frequency of applied voltage pulses for modified capillary, in the frequency range of 10-1000 Hz.

tion during SEE continued after the pulse had been switched off, Fig. 3. Moreover, between every SEE the refilling process inside the capillary takes some time, $\tau_{\mathrm{rf}}$, because the liquid has to return to the equilibrium position. However, the refilling process inside the capillary is very short, as shown

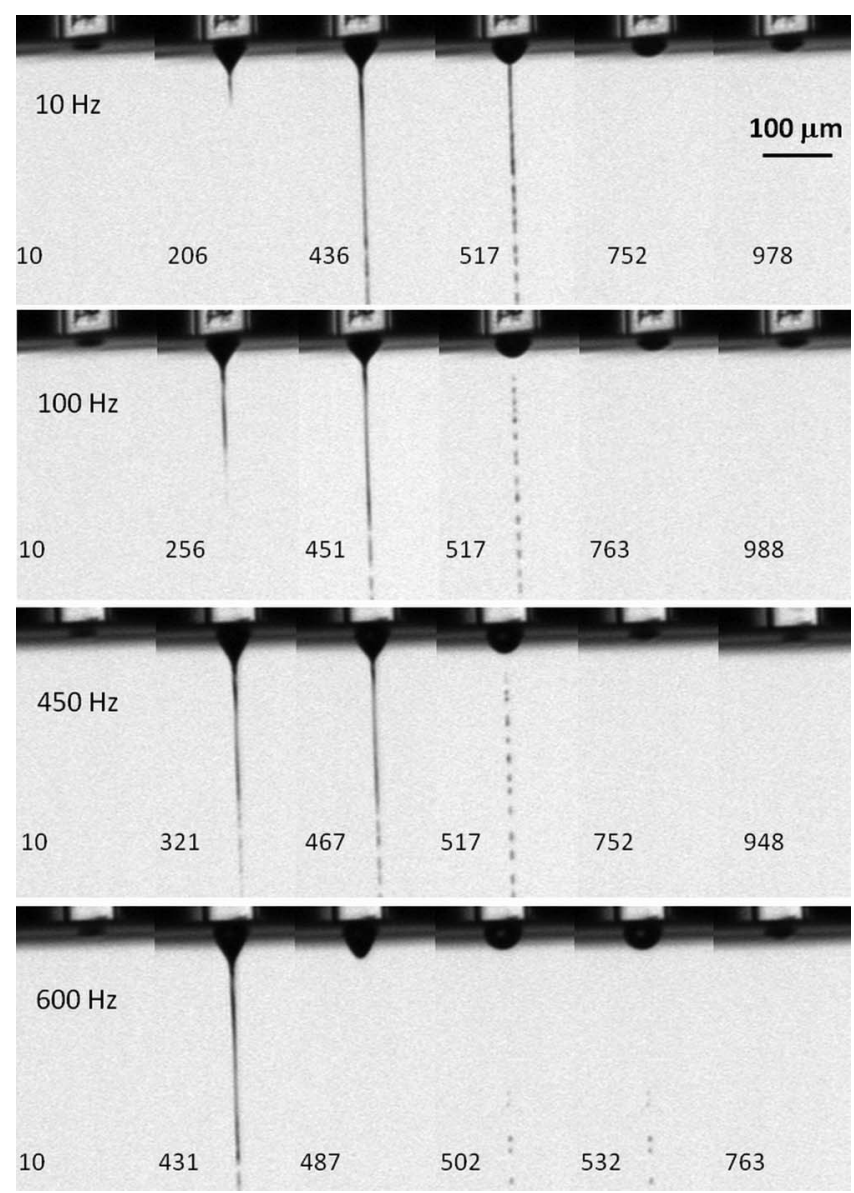

FIG. 3. SEE examples for modified capillary for four different frequencies, For frequency range from 10 to $450 \mathrm{~Hz}$ the ejection is stable and for $600 \mathrm{~Hz}$ the example represents an intermittent ejection. Numbers on the pictures indicate the time (microseconds) when the picture was taken with respect to the beginning of the voltage pulse. The voltage pulse is stopped after $500 \mu \mathrm{s}$. in Table I. The calculated frequency, $f_{\mathrm{c}}$ is $244 \mathrm{~Hz}$. The experimental results in Fig. 2 indicated stable SEEs till $f_{\mathrm{p}(\max )}$ $\sim 450 \mathrm{~Hz}$. The quantitative explanation for the relation between the calculated natural frequency and the observed cutoff frequency for a stable SEE can be given by the known effect that the resonance curve of an under-damped driven oscillation has a maximum close to the natural frequency. The amplitude response in the frequency domain of the forced motion of a mass-spring-damper becomes very low around twice the natural oscillation frequency. Therefore we speculate that SEE above this frequency cannot be stable. At least the results show that the frequency of reproducible SEE is limited by fluid motions in the capillary related to the natural oscillation frequency of the liquid inside the capillary.

This study demonstrated that the hydrodynamic phenomena taking place inside the capillary limits the frequency range of voltage pulses for SEE, when the meniscus/cone size was defined by ID of the nozzle. For the analysis of SEE we used a one-dimensional model describing the displacement and oscillations of the liquid inside the nozzle. The maximum voltage pulse frequency for stable SEE can be defined by analysis of the natural oscillation frequency of the liquid inside the capillary.

This study was supported by the European Research Program, Marie Curie Actions, and Early Stage Fellowship; Project No. MEST-CT-2004-505006 and Philips Research Europe. The authors thank P.A. Barendse, A. Geven, M. Vernhout, and L. van den Besselaar for the technical assistance with the experimental set-up, and D. Burdinski for his help with antiwetting coating.

${ }^{1}$ X. Li, J. Huang, and M. J. Edirisinghe, J. R. Soc., Interface 5, 253 (2008). ${ }^{2}$ W. D. Ristenpart, P. Jiang, M. A. Slowik, C. Punckt, D. A. Saville, and I. A. Aksay, Langmuir 24, 12172 (2008).

${ }^{3}$ R. Moerman, J. Frank, J. C. Marijnissen, T. G. Schalkhammer, and G. W. van Dedem, Anal. Chem. 73, 2183 (2001).

${ }^{4}$ J. U. Park, M. Hardy, S. J. Kang, K. Barton, K. Adair, D. K. Mukhopadhyay, C. Y. Lee, M. S. Strano, A. G. Alleyne, J. G. Georgiadis, P. M. Ferreira, and J. A. Rogers, Nature Mater. 6, 782 (2007).

${ }^{5}$ A. Jaworek and A. T. Sobczyk, J. Electrost. 66, 197 (2008).

${ }^{6}$ M. E. Kuil, J. P. Abrahams, and J. C. M. Marijnissen, Biotechnol. J. 1, 969 (2006).

${ }^{7}$ R. P. A. Hartman, J. P. Borra, D. J. Brunner, J. C. M. Marijnissen, and B. Scarlett, J. Electrost. 47, 143 (1999).

${ }^{8}$ P. K. Notz and O. A. Basaran, J. Colloid Interface Sci. 213, 218 (1999).

${ }^{9}$ W. Balachandran, W. Machowski, and C. N. Ahmad, IEEE Trans. Ind. Appl. 30, 850 (1994).

${ }^{10} \mathrm{Z}$. Huneiti, W. Balachandran, D. Hu, and W. Machowski, Proceedings of the 11th European Conference of ILASS-Europe on Atomization and Sprays, Nürnberg, 1995 (unpublished).

${ }^{11}$ J. Kim, H. Oh, and S. S. Kim, J. Aerosol Sci. 39, 819 (2008).

${ }^{12}$ U. Stachewicz, J. F. Dijksman, C. U. Yurteri, and J. C. M. Marijnissen, Appl. Phys. Lett. 91, 254109 (2007).

${ }^{13}$ U. Stachewicz, J. F. Dijksman, C. U. Yurteri, and J. C. M. Marijnissen, "Volume of liquid deposited per single event electrospraying controlled by nozzle front surface modification," Microfluid. Nanofluid. (to be published).

${ }^{14}$ U. Stachewicz, J. F. Dijksman, D. Burdinski, C. U. Yurteri, and J. C. M. Marijnissen, Langmuir 25, 2540 (2009).

${ }^{15}$ U. Stachewicz, J. F. Dijksman, D. Burdinski, C. U. Yurteri, and J. C. M. Marijnissen, Langmuir 25, 11975 (2009).

${ }^{16} \mathrm{~W}$. T. Thompson, Theory of Vibration with Applications, 2nd ed. (PrenticeHall, Englewood Cliffs, 1981), pp. 1-126. 